\title{
Incubation period for campylobacteriosis and its importance in the estimation of incidence related to travel
}

B J Horn (Beverley.horn@esr.cri.nz) ${ }^{1}$, R J Lake ${ }^{1}$

1. Institute of Environmental Science and Research, Christchurch, New Zealand

Citation style for this article:

Horn BJ, Lake RJ. Incubation period for campylobacteriosis and its importance in the estimation of incidence related to travel. Euro Surveill. $2013 ; 18(40)$ :pii=20602. Available online: http://www.eurosurveillance.org/ViewArticle.aspx?Articleld=20602

Article submitted on 25 September 2012 / published on 03 October 2013

Differentiation between travel-related and domestic cases of infectious disease is important in managing risk. Incubation periods of cases from several outbreaks of campylobacteriosis in Canada, Europe, and the United States with defined exposure time of less than 24 hours were collated to provide information on the incubation period distribution. This distribution was consistent across the varied outbreaks considered, with $84 \%(702 / 832)$ of cases having an incubation period of four days or less and $1 \%$ having an incubation period of eight days or more. The incubation period distribution was incorporated into a model for the number of travel-related cases presenting with symptom onset at given dates after return to their country of residence. Using New Zealand notification data between 2006 and 2010 for cases who had undertaken foreign travel within 10 days prior to symptom onset, we found that $29.6 \%(67 / 227$ cases; $95 \%$ confidence interval ( $\mathrm{Cl}): 28.3-30.8 \%$ ) of these cases were likely to have been domestic cases. When cases with symptom onset prior to arrival were included, the probable domestic cases represented $11.8 \%$ (67/571; $95 \% \mathrm{Cl}: 11.2-12.3 \%)$. Consideration of incubation time distributions and consistent collection of travel start/ end dates with symptom onset dates would assist attribution of cases to foreign travel.

\section{Introduction}

The World Tourism and Travel Council reports that international travel grew in both traveller numbers and economic value for the third consecutive year in 2012 for all regions, including Europe [1]. Therefore differentiation between travel-related and domestic cases of infectious disease is increasingly important to researchers and regulators seeking to understand the epidemiology of disease and potential preventive measures within a single country. The proportion of travel-related bacterial enteric disease cases has been estimated as approximately $20 \%$ for North America and England [2-4], while in Scandinavia and Switzerland the proportion is approximately 50\% [5-7].
To classify a case as infected abroad, the exposure event should have occurred outside the country of residence. Cases that arrive in the country exhibiting symptoms and were out of the country for the entire potential incubation period can be easily categorised. For short visits abroad, it is possible that the infection was acquired domestically before leaving. Cases for which there is a period of time between arrival and symptom onset are more difficult to assign. It is possible that some cases with symptom onset after returning home were actually the result of an exposure in their home country, but with a short incubation period. Thus there is potential for over-reporting of travelacquired illness.

The generally accepted incubation period for campylobacteriosis is two to five days, with a range of one to 10 days [8]. The assignment of a notified case as a travel-related case, or 'out-of-country' acquired infection often depends on the judgement of a doctor or public health worker with knowledge of the incubation period of the pathogen involved. For example, in a Swedish study the clinical notifications were described as infected abroad 'based on the best judgment of the notifying clinician based on patient history and knowledge of the characteristics of the pathogen in question' [5]. The United States (US) FoodNet defines a campylobacteriosis case as foreign-travel related when the patient returned from an international destination within seven days of illness onset [2]. Studies of the importance of travel with regards to acquiring campylobacteriosis using data from the United Kingdom [4,9] and Finland [10] have considered as travel-related campylobacteriosis cases those which had been abroad either five days or two weeks preceding the onset of illness.

In New Zealand, the notified rate of campylobacteriosis in 2011 was 151.9 per 100,000 population (6,692 cases) [11]. Campylobacteriosis risk factor data on notified cases is collated by Public Health Unit (PHU) staff and may include foreign travel risk factor information. This information includes symptom onset date, arrival date 
back in New Zealand, and for some cases the countries visited and duration of the visits. In 2011, of those who reported the information, $7.3 \%$ reported overseas travel during the incubation period. The notification instructions list campylobacteriosis as having the generally accepted incubation period as above.

The aim of this paper is to establish the incubation period profile for campylobacteriosis from defined exposure events reported in the scientific literature, and to use this to model New Zealand notification data to improve estimation of the proportion of cases with symptom onset after arrival, which were infections acquired outside the country.

\section{Methods}

\section{Review of incubation times}

To establish a distribution for the likely incubation period for campylobacteriosis, international outbreak reports from the scientific literature located by keyword searches in PubMed 'campy* AND outbreak' and Science Direct 'campylobacter AND outbreak' were reviewed. The records were reviewed by title, abstract, and if necessary full text, to identify outbreaks of campylobacteriosis (only) which could be associated with a fixed event, and where the event started and finished within a 24 hour period. Examples included sporting and entertainment events. Outbreaks associated with contaminated drinking water or ongoing food supplies were not included, as it was not possible to define the exact exposure date for cases associated with this type of outbreak.

Reports from outbreaks with fixed exposure dates were then reviewed for information on the period between exposure event (day o) and the onset of symptoms. This provides the incubation time in days for campylobacteriosis symptoms to appear.

For some outbreaks the time to symptom onset is given for both laboratory-confirmed and suspected (probable) cases. For the purposes of this paper, we assumed all suspected cases that occurred within the time range of the occurrence of confirmed cases were also due to the exposure associated with the outbreak.

The periods between exposure and symptom onset retrieved from the individual outbreak reports were examined graphically to compare the distribution of incubation periods for consistency across different events. We then combined the cases from the outbreaks and compiled a data set including all the cases with known period between exposure and symptom onset. This dataset was used to establish the distribution of incubation times. For the proportion of cases with given incubation periods (days), 95\% confidence intervals $(\mathrm{Cl})$ were estimated from the 2.5 th and 97.5 th percentiles of 5,000 bootstrap samples taken with replacement. Analyses were conducted using the R statistical package [12].
Review of New Zealand notification data Notification data are recorded using a web-based application (EpiSurv) available to staff at each of the 18 PHUs in New Zealand. These data are transferred to the Institute of Environmental Science and Research (ESR) Ltd., where they are collated, analysed and reported on behalf of the Ministry of Health.

Anonymised notification reports of cases of campylobacteriosis for which foreign travel was reported as a risk factor were obtained from EpiSurv for the period 2006 to 2010, following approval from the MultiRegion Ethics Committee. These records were carefully reviewed to identify a subset of the cases which reported both the date of arrival in New Zealand and the date of onset of symptoms which was either during travelling or after return to New Zealand. The time in days relative to arrival in New Zealand was calculated for each case, with the arrival date set to day zero. While there are fields for the dates of arrival to and departure from countries visited in the notification database, these fields are rarely filled in and so were not used in this study.

The incubation period distribution derived from the combined outbreak dataset was used to estimate the proportion of travel-related cases with symptoms starting on each day in the range of one to 10 days after returning to New Zealand that could be due to domestically-acquired infection. It was assumed that cases were equally likely to have been infected on any of the 10 days prior to arriving in New Zealand, and there was no difference in the distribution of the incubation period for infections acquired domestically and abroad. Given these assumptions, the estimated number of cases associated with travel abroad with onset on day $d$ after arrival in New Zealand, $C_{T}$, can be given by,

$C_{T}(d)=A\left(1-\sum_{i=1}^{d}\right.$ Proportion of cases with incubation period $\left.i\right)$.

The scaling factor, $A$, was derived from fitting the equation to the number of cases recording symptom onset one day after return to New Zealand and the proportion of cases with incubation period $i$ was taken from the outbreak data distribution.

The number of estimated foreign travel-related cases was compared graphically to the number of notified cases associated with foreign travel to determine the number of days after arrival in New Zealand after which any consistent difference in the two estimates could be observed. From this point, the difference between the notifications and the model outputs for the remaining days up to 10 days post arrival in New Zealand was used to estimate the over-prediction of travel-acquired infections. The estimated over-prediction of travelacquired infections were presented as the 2.5th, 50th and $97.5^{\text {th }}$ percentiles of over-prediction calculated 
Campylobacteriosis outbreaks $(n=7)$ with reported incubation periods for cases $(n=832)$ and defined exposure events, 1983-2010

\begin{tabular}{|c|c|c|c|}
\hline Exposure & $\begin{array}{l}\text { Number of campylobacteriosis } \\
\text { cases with incubation data }\end{array}$ & Country & Reference \\
\hline Orienteering event with raw milk drinks available & 451 & Switzerland & Stalder et al. 1983 [13] \\
\hline Mud ingestion during mountain bike event & 225 & Canada & Stuart et al. $2010[14]$ \\
\hline Cadets killing, cooking and eating chickens & 78 & Netherlands & Brouwer et al. 1979 [15] \\
\hline Wedding meal & 24 & England & Inns et al. 2010 [16] \\
\hline Farm visit including drinking unpasteurised milk & 23 & Wales & Evans et al. 1996 [17] \\
\hline Dinner dance & 20 & England & Skirrow et al. $1981[18]$ \\
\hline Undercooked barbequed chicken & 11 & United States & Istre et al. 1984 [19] \\
\hline
\end{tabular}

from 5,000 bootstrap samples of the incubation period distribution taken with replacement.

\section{Results}

\section{Incubation period distribution}

From the PubMed (641 records) and Science Direct ( 85 records) searches, seven outbreaks with known distinct exposure events and number of reported cases for each incubation period (days) were identified. These are summarised in the Table. Laboratory-confirmed Campylobacter jejuni was reported as being associated with all the outbreaks. The three largest outbreaks each involved between 78 and 451 cases [13-15] and provided incubation periods for a total of 754 cases while four smaller outbreaks [16-19] involved 11 to 24 cases per outbreak and provided a total of 78 incubation periods.

The incubation period distribution is presented in Figure 1a for the three large outbreaks and Figure $1 \mathrm{~b}$ for the smaller outbreaks. The incubation period distribution is displayed as the cumulative proportion of the cases in each outbreak to have an incubation period of equal to or less than the days given. The distribution curves are similar for the large and smaller outbreaks.

Figure 2 shows the distribution of incubation period for cases from all outbreaks for up to 10 days. Of the 832 cases with known exposure and symptom onset dates, only $17(1 \%)$ recorded an incubation period of greater than seven days. Most cases, 702 (84\%), had an incubation period of four days or less.

\section{Overseas travel case attribution}

A total of 945 confirmed New Zealand cases of campylobacteriosis with overseas travel as a reported risk factor over the period 2006 to 2010 were obtained from EpiSurv records. Of these, 121 (13\%) did not have an exact symptom onset date and of the remaining records $253(27 \%)$ did not have an exact arrival date in New Zealand. These cases were excluded from the analysis, resulting in 571 records $(60 \%)$ where the number of days between arrival in New Zealand and symptom onset could be calculated. For 344 of these cases, symptoms had occurred prior to arrival in New Zealand, leaving 227 cases with a defined period to symptom onset of one to 10 days after arrival.

Figure 3 shows the frequency of cases' symptom onset relative to the number of days after arrival in New Zealand. Most cases (66\%) record symptom onset within one to four days, but for longer periods the frequency is higher than might be expected from the distribution shown in Figure 2.

The incubation period distribution in Figure 2 was used to derive a likely frequency distribution for symptom onset following arrival in New Zealand. The scaling factor for this data, A, was derived to be 69.5 cases, which resulted in a predicted frequency of cases which acquired campylobacteriosis abroad, also shown in Figure 3.

Comparison of the predicted number of cases associated with foreign travel, with the number of cases reporting foreign travel as a risk factor in notification records (Figure 3) suggests that a proportion of the cases with symptom onset of five or more days after arrival in New Zealand could be domestic cases. Using the subset of travel-associated cases with symptom onset after return to New Zealand, this proportion with domestically-acquired campylobacteriosis is $67 / 227$ (29.6\%, $95 \% \mathrm{Cl}: 28.3-30.8 \%)$ of cases. A lower bound for the overestimation of all travel-associated cases, $67 / 571$ (11.8\%, 95\% Cl: $11.2-12.3 \%$ ), can be calculated by assuming that all infections with symptom onset before arrival were acquired overseas. However, this overestimation percentage will be higher if some of the cases became infected before the start of their travels. 


\section{FIGURE 1}

Cumulative density plots for campylobacteriosis incubation periods of up to 10 days for (a) three large campylobacteriosis outbreaks along with the distribution obtained from combining the data from four smaller outbreaks, and (b) the four smaller outbreaks, 1983-2010 $(\mathrm{n}=832$ cases $)$

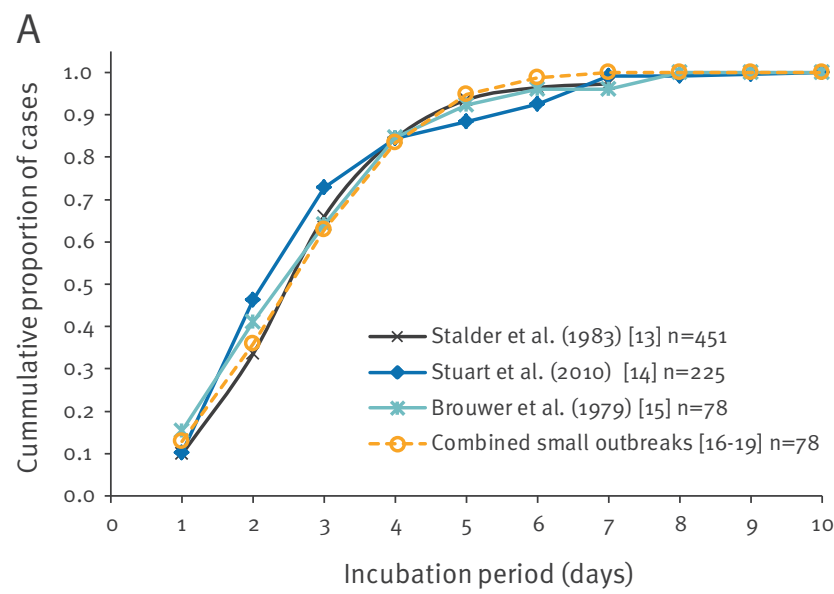

B

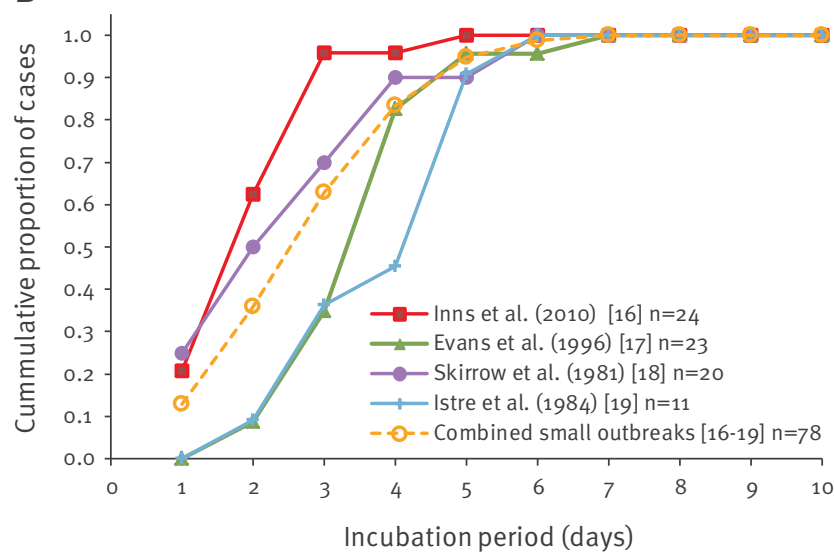

\section{FIGURE 2}

Distribution of the proportion of campylobacteriosis cases in function of their incubation periods among 832 cases associated with outbreaks with known exposure events

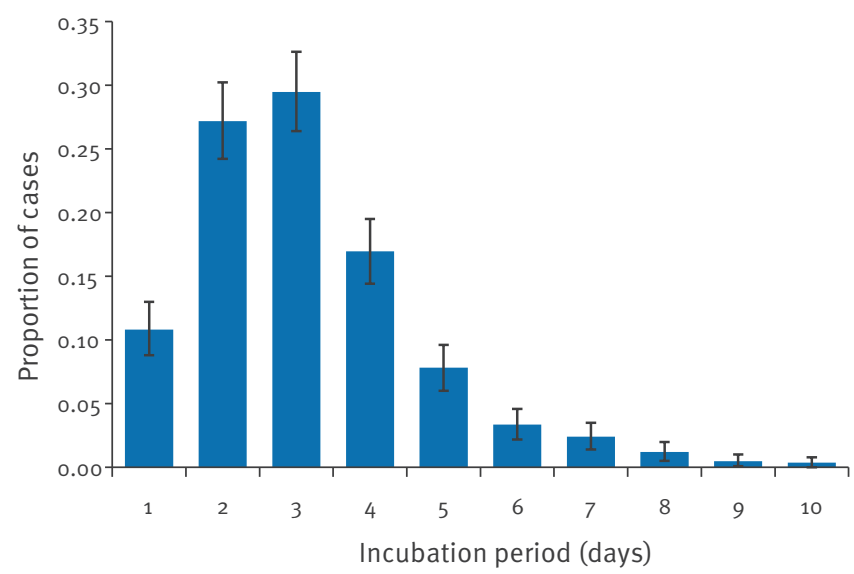

2.5th and 97.5th percentile bootstrap intervals of the proportion are plotted.

\section{Discussion}

This analysis suggests the importance of travel as a risk factor may be overestimated when only 'yes/no' answers to foreign travel in the incubation period data from notification records are considered. This is a minor adjustment for New Zealand, where travel is estimated to be a risk factor in less than $10 \%$ of notified cases of campylobacteriosis [11]. However, in Europe and North America where travel-related cases are a higher proportion of the total reported, and where out of country travel may be for shorter periods, the adjustment required to attribution estimates, based on our results, may be more significant.

The analysis depends on reported outbreak incubation period data. The three largest outbreaks considered in this study have similar distributions for incubation period, with nearly half the cases showing symptoms within two days of the exposure event and $85 \%$ of cases reporting symptoms starting in the first four days after the exposure event. The four smaller outbreaks show more variation than the larger outbreaks. The wedding meal outbreak [16] has a larger number of cases with short incubation periods ( $\leq 3$ days) while the farm visit [17] and the barbeque outbreak [19] have a smaller proportion of cases showing symptoms over this time period. Such variation may be strain dependent or due to the natural variation expected from sampling smaller datasets. Combining the data from the four smaller datasets provides a distribution close to those given by the three larger datasets as shown in Figure 1a.

In the outbreaks considered, only 18 of the 832 cases had an incubation period of more than seven days. In the outbreak reported by Evans et al [17], all 23 primary cases had symptom onset within seven days after the farm visit. However, a number of secondary cases were observed from day seven onwards, which were family members of cases who did not take part in the farm visit. It is possible that notified or outbreak cases with incubation periods of eight to 10 days do not all result from the identified exposure, but are actually secondary cases.

In this analysis we have assumed that all strains of Campylobacter have the same incubation period pattern, which is supported by the consistency of the outbreak data. Studies in Switzerland and New Zealand have found differing genotypes of Campylobacter in domestic and travel-associated cases $[7,20]$. We also assume the incubation period pattern found in the various countries listed in the Table where outbreaks occurred also applies to the New Zealand population.

A recent analysis of gastrointestinal infections for Norway found that of those reported as travel-associated and with symptom onset after travel return, 94\% of campylobacteriosis cases occurred within the commonly reported incubation period ( $\leq 5$ days), and over $98 \%$ occurred within the maximum incubation period 


\section{FIGURE 3}

Comparison of the predicted number of travel-related cases with a subset of the recorded numbers of cases of campylobacteriosis, with symptom onset within 10 days following return to New Zealand, 2006-2010 (subset $\mathrm{n}=227$ )

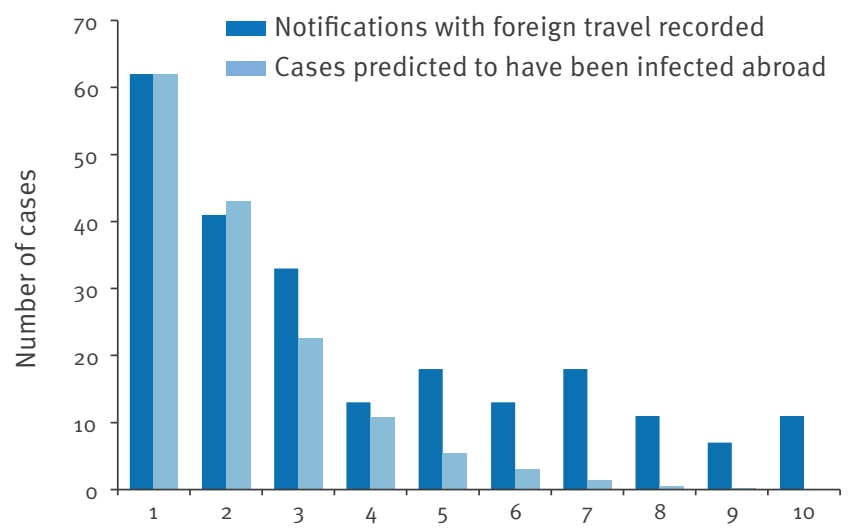

Day of symptom onset after arrival in New Zealand

( $\leq 10$ days) [6]. Our analysis of outbreak data is in agreement with these results ( $92 \%$ of cases with an incubation period $\leq 5$ days).

Data on arrival and symptom onset dates were essential for this type of analysis, and enabled careful filtering of the records. As was also found in the analysis of cases from Norway, data for return date and date of symptom onset were complete for only approximately $60 \%$ of New Zealand cases which may have been travel associated [6]. We concur with those authors in recommending more complete reporting of travel data, in particular date of departure and duration of travel, as exposures prior to departure may be important for very short trips (less than 4-5 days) combined with longer incubation periods. Such information should be included in the risk factor information collated as part of case investigations.

Although the adjustment for travel-associated cases examined in this paper will not identify specific cases that could be domestically acquired, it would improve estimation of the incidence of domestically-acquired infections, and hence burden of disease. We recommend a close examination of the incubation period distribution as part of future estimates of the attribution of diseases to foreign travel.
References

1. World Travel and Tourism Council (WTTC). Travel \& Tourism Economic Impact 2013 Europe. London: WTTC. Available from: http://www.wttc.org/site_media/uploads/downloads/ europe2013_1.pdf

2. Kendall ME, Crim S, Fullerton K, Han P V, Cronquist A B, Shiferaw B, et al. Travel-associated enteric infections diagnosed after return to the United States, Foodborne Diseases Active Surveillance Network (FoodNet), 20042009. Clin Infect Dis. 2012;54 Suppl 5:S480-7. http://dx.doi. org/10.1093/cid/cis052. PMid:22572673.

3. Ravel A, Nesbitt A, Marshall B, Sittler N, Pollari F. Description and burden of travel-related cases caused by enteropathogens reported in a Canadian community. J Travel Med. 2011;18(1):819. http://dx.doi.org/10.1111/j.1708-8305.2010.00471.x. PMid:21199137.

4. Zenner D, Gillespie I. Travel-associated salmonella and campylobacter gastroenteritis in England: estimation of underascertainment through national laboratory surveillance. J Travel Med. 2011;18(6): 414-7. http://dx.doi.org/10.1111/j.17088305.2011.00553.x. PMid:22017719.

5. Ekdahl K, Andersson Y. Regional risks and seasonality in travel-associated campylobacteriosis. BMC Infect Dis. 2004;4(1):54. http://dx.doi.org/10.1186/1471-2334-4-54. PMid:15569393. PMCid:PMC539239.

6. Guzman-Herrador B, Vold L, Nygard K. Surveillance of travel-associated gastrointestinal infections in Norway, 2009-2010: are they all actually imported? Euro Surveill. 2012;17(41): pii=20294. Available from: http://www. eurosurveillance.org/ViewArticle.aspx?Articleld=20294 PMid:23078812.

7. Niederer L, Kuhnert P, Egger R, Büttner S, Hächler H, Korczak BM. Genotypes and antibiotic resistances of Campylobacter jejuni and Campylobacter coli isolates from domestic and travel-associated human cases. Appl Environ Microbiol. 2012;78(1): 288-91. http://dx.doi.org/10.1128/AEM.06194-11. PMid:22020515. PMCid:PMC3255622.

8. Campylobacter enteritis. In: Heymann D, editor. Control of communicable diseases manual 18th edition. Washington DC: American Public Health Association; 2004.

9. Campylobacter Sentinel Surveillance Scheme. Foreign and domestic travel and the risk of Campylobacter infection: results from a population-based sentinel surveillance scheme. J Travel Med. 2003;10(2):136-8.

10. Hakanen A, Jousimies-Somer H, Siitonen A, Huovinen P, Kotilainen P. Fluoroquinolone resistance in Campylobacter jejuni isolates in travelers returning to Finland: association of ciprofloxacin resistance to travel destination. Emerg Infect Dis. 2003:9(2):267-70. http://dx.doi.org/10.3201/eido902.020227. PMid:12604004. PMCid:PMC2901943.

11. Institute of Environmental Science and Research Ltd (ESR). Notifiable and Other Diseases in New Zealand: Annual Report 2011. Porirua: ESR; Apr 2012, Updated Oct 2012. [Accessed 1 May 2013]. Available from: http://www.surv.esr.cri.nz/ PDF_surveillance/AnnualRpt/AnnualSurv/2011/2011AnnualSur vRpt.pdf

12. R Development Core Team. R: A language and environment for statistical computing. Vienna: R Foundation for Statistical Computing. [Accessed 1 May 2013]. Available from: http:// www.R-project.org/

13. Stalder H, Isler R, Stutz W, Salfinger M, Lauwers S, Vischer W. [Contribution to the epidemiology of Campylobacter jejuni. From asymptomatic excretion by a cow in the cowshed to overt disease in over 500 persons]. Schweiz Med Wochenschr. 1983;113(7):245-9. German. PMid:6836250.

14. Stuart TL, Sandhu J, Stirling R, Corder J, Ellis A, Misa $\mathrm{P}$, et al. Campylobacteriosis outbreak associated with ingestion of mud during a mountain bike race. Epidemiol Infect. 2010;138(12):1695-703. http://dx.doi.org/10.1017/ So95026881000049X. PMid:20334726.

15. Brouwer R, Mertens MJ, Siem TH, Katchaki J. An explosive outbreak of Campylobacter enteritis in soldiers. Antonie Van Leeuwenhoek. 1979;45(3):517-9. http://dx.doi.org/10.1007/ BFo0443293. PMid:576030.

16. Inns T, Foster K, Gorton R. Cohort study of a campylobacteriosis outbreak associated with chicken liver parfait, United Kingdom, June 2010. Euro Surveill. 2010;15(44):pii=19704. Available from: http://www. eurosurveillance.org/ViewArticle.aspx?Articleld=19704 PMid:21087588.

17. Evans MR, Roberts RJ, Ribeiro CD, Gardner D, Kembrey D. A milk-borne Campylobacter outbreak following an educational farm visit. Epidemiol Infect. 1996;117(3):457-62. http:// dx.doi.org/10.1017/So950268800059112. PMid:8972669. PMCid:PMC2271642. 
18. Skirrow MB, Fidoe RG, Jones DM. An outbreak of presumptive food-borne campylobacter enteritis. J Infect. 1981;3(3):234-6. http://dx.doi.org/10.1016/S0163-4453(81)90819-7

19. Istre GR, Blaser MJ, Shillam P, Hopkins RS. Campylobacter enteritis associated with undercooked barbecued chicken. Am J Public Health. 1984;74(11):1265-7. http://dx.doi.org/10.2105/ AJPH.74.11.1265. PMid:6496822. PMCid:PMC1652037.

20. Gilpin BJ, Walsh G, On SL, Smith D, Marshall JC, French NP. Application of molecular epidemiology to understanding campylobacteriosis in the Canterbury region of New Zealand. Epidemiol Infect. 2013 Jun;141(6):1253-66. http://dx.doi. org/10.1017/S0950268812001719. PMid:22906314. 